\title{
Epidemiology and resistance trends of Staphylococcus aureus isolated from vaginal samples: a 10-year retrospective study in Hungary
}

\author{
Márió Gajdács ${ }^{1,2}$, Edit Urbán³
}

\begin{abstract}
Introduction: The vaginal flora is a complex microbial environment. The disruption of this niche usually leads to a pathological state and symptoms in patients. Aerobic vaginitis is a distinct form of vaginal inflammation, mainly caused by aerobic/facultative anaerobic bacteria (Staphylococcus aureus, Streptococcus agalactiae, and members of the Enterobacteriaceae). This study describes the prevalence and antibiotic susceptibility patterns of $S$. aureus isolated from vaginal samples from females at a tertiarycare teaching hospital in Hungary.

Methods: This retrospective study was carried out using data collected at the Albert Szent-Györgyi Clinical Center (University of Szeged) corresponding to a 10-year period (2008-2017). Antimicrobial susceptibility testing was performed using the disk diffusion method and gradient diffusion, using EUCAST interpretative standards. Methicillin-resistant S. aureus (MRSA) was detected on mannitol salt agar using cefoxitin disks, the PBP2' Latex Agglutination Test Kit, and matrix-assisted laser desorption/ionization time-of-flight (MALDI-TOF) mass spectrometry.

Results: The median age of affected patients was 31 years. Most (93.95\%) of the samples received were vaginal swabs. A total of 3,356 individual isolates were recorded (335.6 \pm 89.10/year, range: $213-480$ isolates). In $91.4 \%$ of samples, S. aureus was the only pathogen isolated. The highest levels of resistance were detected against erythromycin (11.11 $\pm 3.65 \%$, range: $6.76-17.17 \%)$ and clindamycin ( $10.85 \pm 3.36 \%$, range: $6.49-15.54 \%$ ), whereas resistance rates against doxycycline, ciprofloxacin, chloramphenicol, sulfamethoxazole-trimethoprim, and gentamicin were much lower (0-4.48\%). Susceptibility to cefoxitin was observed in $97.79 \%$ of the isolates; 74 strains were MRSA. All MRSA strains were susceptible to the antibiotics used for therapy for multidrug-resistant Gram-positive infections.

Conclusions: A slow and steady increase in resistance levels could be observed (mainly corresponding to MRSA isolates). Although the present resistance trends are still advantageous (compared to European resistance levels) and do not hinder adequate therapy, continuous surveillance of resistance levels is recommended. Macrolides and clindamycin should be used with caution, and, if available, only when susceptibility to these drugs has been verified.
\end{abstract}

Keywords: Staphylococcus aureus, epidemiology, antimicrobial resistance, vaginal samples

Received: 3 June 2019| Returned for modification: 6 August 2019 | Accepted: 9 September 2019

\section{Introduction}

The vaginal flora is a complex microbial environment, consisting of a multitude of microbial species in variable quantities and proportions $(1,2)$. This collection of microorganisms is very distinct from other anatomical regions of the human body. In premenarcheal girls, Lactobacillus species are infrequent or minor constituents of the vaginal microbiota, with other facultative and strict anaerobes being in abundance. This corresponds to a neutral or slightly alkaline vaginal $\mathrm{pH}$ during early childhood. In healthy adult women, the microbial diversity is greatly reduced, with an abundance of Lactobacillus species, responsible for the acidification (due to lactic acid and $\mathrm{H}_{2} \mathrm{O}_{2}$ production) of this environment $(3,4)$. The colonization of the vaginal flora by lactobacilli is influenced by their adhesion levels to vaginal epithelial cells; in addition, some of the nutrients are derived from these dead epithelial cells of the female hosts (and others can be found in some secretions of the glands in the lower reproductive tract) $(5,6)$. Following puberty, the thicker stratified epithelium and higher levels of glycogen in the reproductive tract of females also favor Lactobacillus colonization (2-4). In addition to lactobacilli, other microorganisms commonly identified as commensals in the female genital tract include Staphylococcus aureus, Streptococcus agalactiae (group B Streptococcus), Enterococcus faecalis, Escherichia coli, and Candida albicans (7-9). After menopause, estrogen levels drop in the female body, dramatically affecting the vaginal microbiota; the $\mathrm{pH}$ rises to 6.0 to 8.0, corresponding to the reduction or elimination of lactobacilli and the increased colonization of the vaginal tract by members of the Enterobacteriaceae family, Gram-positive cocci, and Bacteroides and Prevotella species (fecal flora) (2-6). However, if the normal vaginal flora is disrupted, these bacteria become pathogenic, causing vaginal discharge (often with a putrid odor) and other symptoms such as itching, which is detrimental to patients' quality of life (QoL) $(10,11)$. If there is a multitude of bacterial species present in the vaginal flora, that usually corresponds to a disrupted, pathological state of the vaginal microbiome.

Aerobic vaginitis (AV) was first characterized in 2002 as a distinct form of vaginal inflammation from bacterial vaginosis (BV) (12-14). Although both conditions are characterized by the disruption of the female genital Lactobacillus flora, BV is a non-inflammatory condition, distinguished by the high quantity of anaerobic bacteria, whereas in AV there is significant inflammation and it is mainly caused by aerobic/facultative anaerobic bacteria (13-14). 
The main causes of $\mathrm{AV}$ include overpopulation of the vaginal flora with S. aureus, S. agalactiae, or E. coli, typically concurring with an increased inflammatory response or epithelial atrophy (or both) (15). The diagnosis of AV should be performed on the basis of wet mount microscopy, and it should be differentiated from BV on the basis of Nugent's method or Gram-staining $(12-13,16)$. Antimicrobial susceptibility testing results aid the choice of the appropriate antibiotic; however, therapy on the basis of culture results only is not recommended. The prevalence of $\mathrm{AV}$ is around 8 to $11 \%$ in pregnant women, and 5 to $24 \%$ in women reporting complaints regarding the reproductive system (12-17).

Infections of the vagina have been associated with a significantly increased risk of preterm labor and low birth weight; in addition, other sequela may include pelvic inflammatory disease (PID), which can lead to ectopic pregnancies, tubal infertility, and other dysfunctions of the female reproductive system $(18,19)$. The role of bacterial infections in the vagina has also been described in the progression of cervical dysplasia and the transmission/acquisition of various viral infections (HIV and herpes simplex virus 2) (20).

The epidemiology of genitourinary pathogens varies significantly with respect to the healthcare institution in question and the geographical localization (21). The emerging threat of methicillin-resistant $S$. aureus (MRSA) infections is a grave concern for clinicians (22). This study describes the prevalence and antibiotic susceptibility patterns of $S$. aureus, a significant pathogen in AV, isolated from vaginal samples at a tertiary-care teaching hospital in Hungary during a 10-year study period (2008-2017).

\section{Methods}

\section{Study design, data collection}

This retrospective study was carried out using data collected between January 1st, 2008 and December 31st, 2017 at the microbiology laboratory of the Albert Szent-Györgyi Clinical Center. This teaching hospital annually serves more than 400,000 patients in the Southern Great Plain of Hungary, according to the national insurance data (23). An electronic search in the records of the MedBakter laboratory information system (LIS) for samples processed at our laboratory that were positive for $S$. aureus was conducted by the authors.

For the purposes of data analyses, the 10-year study period was divided into two 5-year periods (2008-2012 and 2013-2017, respectively). In the data analysis, the authors included samples with 105 or more colony-forming units (CFU) for $S$. aureus (except in cases where international guidelines recommend otherwise). Only the first isolate per patient was included in the study. In addition, $S$. aureus isolates with divergent susceptibility patterns were considered different isolates. In addition, patient data were collected regarding demographic characteristics (age and reason for sample submission as indicated on the request forms for microbiological analysis). The study was deemed exempt from an ethics review by the Institutional Review Board and informed consent was not required because data anonymity was maintained.

\section{Identification of relevant isolates}

Sample processing was carried out according to guidelines in routine clinical bacteriology. All culture media (5\% sheep blood agar, chocolate agar, and eosin methylene blue agar) were incubated at $37^{\circ} \mathrm{C}$ for 24 to 48 hours in a $5 \% \mathrm{CO}_{2}$ atmosphere. If $S$. aureus was detected from the relevant samples, the plates were passed on for further processing. Between 2008 and 2012, presumptive phenotypic (biochemical reaction-based) methods and VITEK 2 ID (bioMérieux, Marcy-l'Étoile, France) were used for bacterial identification, whereas after 2013 this was complemented by matrix-assisted laser desorption/ionization time-of-flight mass spectrometry (MALDI-TOF MS; Bruker Daltonik GmbH, Germany) (24). The methodology of sample preparation for MALDI-TOF MS measurements was described elsewhere (25).

\section{Antimicrobial susceptibility testing}

Antimicrobial susceptibility testing (AST) was performed using the Kirby-Bauer disk diffusion method and, when appropriate, E-test (Liofilchem, Abruzzo, Italy) on Mueller-Hinton agar (MHA) plates. In addition, for the verification of discrepant results, VITEK 2 AST (bioMérieux, Marcy-l'Étoile, France) was also utilized. The interpretation of the results was based on EUCAST breakpoints (http://www.eucast.org). S. aureus ATCC 29213 and S. aureus ATCC 43300 were used as quality control strains.

To evaluate the resistance trends of isolated strains, erythromycin (ERI), chloramphenicol (CHL), ciprofloxacin (CIP), clindamycin (CLI), doxycycline (DOX), gentamicin (GEN), and sulfamethoxazole/trimethoprim (SXT) were chosen as indicator antibiotics, based on the local antibiotic utilization data method). MRSA was detected using mannitol salt agar (MSA) using cefoxitin (FOX) disks ( $<22 \mathrm{~mm}$ zone diameter) and PBP2' Latex Agglutination Test Kit (Thermo Fisher Scientific Hungary GmbH, Budapest, Hungary). After 2013, a combined MALDI-TOF MS and PBP2' latex agglutination protocol was introduced in our laboratory (26). MRSA-positive isolates were considered to be resistant to all $\beta$-lactam antibiotics. In the case of MRSA-positivity, susceptibility testing for additional antibiotics (vancomycin, VAN; linezolid, LZD; daptomycin, DAP; mupirocin, MUP; and fusidic acid, FZA) was performed (22). During data analysis, intermediate results were grouped with and reported as resistant. Inducible CLI resistance was detected using the D test, and these strains were also reported as resistant.

\section{Statistical analysis}

Descriptive statistical analysis (including means or medians with ranges and percentages to characterize data) was performed using Microsoft Excel 2013 (Microsoft Corp., Redmond, WA). Statistical analyses were performed with SPSS software version 24 (IBM SPSS Statistics for Windows 24.0, IBM Corp., Armonk, NY), using the $x^{2}$ test, Student's $t$-test, and Mann-Whitney U test. The normality of variables was tested using Shapiro-Wilk tests. $P$ values < 0.05 were considered statistically significant.

\section{Results}

\section{Demographic characteristics, sample types}

The median age of affected patients was 31 years in both the first (2008-2012) and second (2013-2017) half of the study period (range 2008-2012: 2-91 years, range 2013-2017: 9-83 years; $p>0.05$ ), and the detailed age distribution of patients is presented in Figure 1. Most (93.95\%) of the samples received were vaginal swabs, and $5.62 \%$ were high cervical swabs, $0.29 \%$ were explanted intrauterine devices (IUDs), and $0.14 \%$ were urethral swabs.

The main indications for the sample submission associated 
with samples positive for $S$. aureus included vaginitis/vulvitis (49.79\%) and high-risk pregnancy (31.02\%); less common indications included nonspecific abdominal pain (1.73\%), symptoms of menopause (1.31\%), suspicion of a urinary tract infection (0.66\%), unspecified pathology of the cervix (0.39\%), amenorrhea/dysmenorrhea $(0.33 \%)$, polycystic ovary syndrome (PCOS) or other cysts of the female genitourinary tract $(0.30 \%)$, endometriosis $(0.30 \%)$, or other nonspecific reasons (14.84\%).

\section{Distribution of $S$. aureus isolates among vaginal samples from women}

During the 10-year surveillance period (January 1st, 2008 - December 31st, 2017), the Institute of Clinical Microbiology received 4,012 samples from outpatient clinics and inpatient departments that turned out to be positive for $S$. aureus; after data consolidation, 3,356 individual isolates were recorded (335.6 \pm 89.10/year, range: $213-480$ isolates; highest in 2016, lowest in 2008) during the study period (the detailed isolation frequency is presented in Fig. 2). A considerable but not significant increase $(p=0.182)$ was observed in the isolation frequency in the second part of the study period ( $296.80 \pm 58.81$ vs. $374.40 \pm 103.15)$. In $91.4 \%$ of samples, $S$. aureus was the only pathogen isolated.

\section{Antibiotic susceptibility trends among $\boldsymbol{S}$. aureus strains in the study period}

The resistance levels of the individual $S$. aureus isolates are summarized in Table 1. The highest levels of resistance were detected

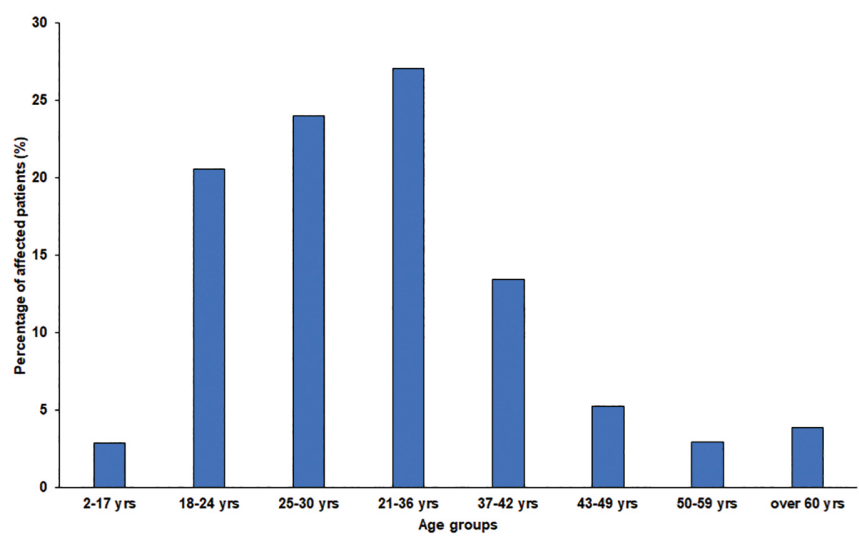

Figure 1 | Age distribution of affected patients during study period. against ERI (11.11 $\pm 3.65 \%$, range: $6.76-17.17 \%)$ and CLI (10.85 \pm $3.36 \%$, range: $6.49-15.54 \%$ ), whereas resistance rates against DOX $(2.19 \pm 2.10 \%$, range: $0-4.48 \%)$, CIP $(1.50 \pm 1.04 \%$, range: o-3.41\%), CHL (0.67 $\pm 0.40 \%$, range: $0-1.90 \%)$, SXT (0.31 $\pm 0.81 \%$, range: $0-1.61 \%)$, and GEN (0.20 $\pm 0.14 \%$, range: $0-0.59 \%)$ were much lower in comparison. Statistically significant differences during the two halves of the study period could only be observed in the case of CLI ( $p<0.001)$, and no such difference was noted for other antibiotics $(p=0.138, p=0.685, p=0.345, p=0.147, p$ $=0.693$, and $p=0.292$ for ERI, CHL, CIP, DOX, GEN, and SXT, respectively). Susceptibility to FOX was observed in $97.79 \%$ of the isolates over the 10-year study period.

Overall, 74 strains were detected that were MRSA-positive (FOX resistant and positive for $\mathrm{PBP}_{2}$ latex agglutination; the first isolate was recovered in 2012), representing $2.21 \pm 1.91 \%$ of the isolates (range $0-5.28 \%$, highest in 2017). In addition to MRSA-positivity, $56(75.7 \%)$ strains were also resistant to ERI, 38 (51.4\%) to CLI, $31(41.9 \%)$ to DOX, $11(14.9 \%)$ to CIP, and 6 (8.1\%) to GEN. No MRSA-positive strains were resistant to CHL and SXT; in addition, all MRSA strains were susceptible to the antibiotics specifically used for the therapy for MDR Gram-positive infections or MRSAdecolonization (namely VAN, LZD, DAP, MUP, and FZA).

\section{Discussion}

This study reports on the epidemiological trends and resistance levels of $S$. aureus in gynecological samples in the Southern Great Plain of Hungary over a long surveillance period (10 years). To date, this is the first and longest such study in Hungary. There are

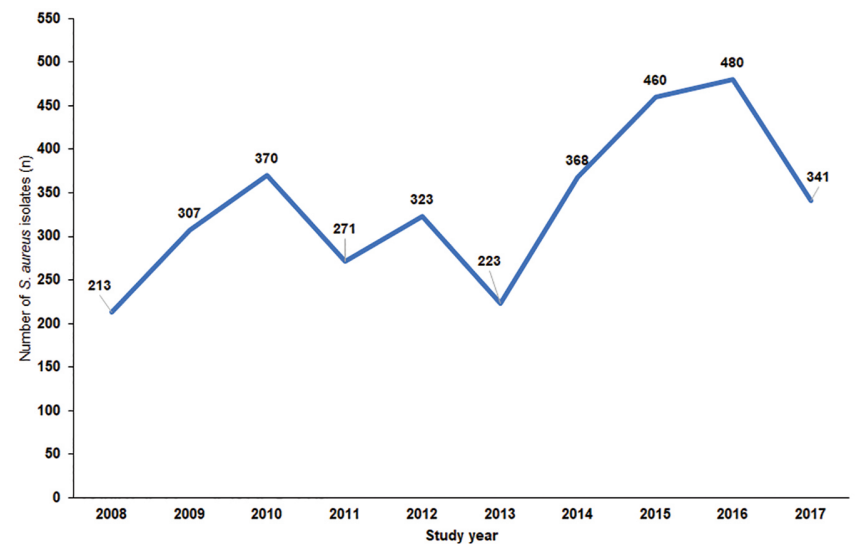

Figure 2 | Frequency of Staphylococcus aureus isolates in vaginal samples from outpatients and inpatients during the 10-year study period.

Table 1 | Table 1. Percentage of $S$. aureus resistant isolates to the tested antibiotics.

\begin{tabular}{|c|c|c|c|c|c|c|c|c|}
\hline \multirow[b]{2}{*}{ Study year } & \multicolumn{8}{|c|}{ Antibiotics } \\
\hline & ERI & $\mathrm{CHL}$ & $\mathrm{CIP}$ & CLI & DOX & GEN & SXT & MRSA* \\
\hline 2008 & $8.45 \%$ & $0.00 \%$ & $0.00 \%$ & $7.98 \%$ & $6.10 \%$ & $0.00 \%$ & $0.00 \%$ & $0.00 \%$ \\
\hline 2009 & $11.40 \%$ & $0.65 \%$ & $1.63 \%$ & $9.45 \%$ & $0.65 \%$ & $0.33 \%$ & $2.61 \%$ & $0.00 \%$ \\
\hline 2010 & $6.76 \%$ & $0.54 \%$ & $0.27 \%$ & $6.49 \%$ & $2.43 \%$ & $0.54 \%$ & $0.27 \%$ & $0.00 \%$ \\
\hline 2011 & $8.86 \%$ & $0.74 \%$ & $0.74 \%$ & $8.12 \%$ & $2.95 \%$ & $0.00 \%$ & $0.37 \%$ & $0.00 \%$ \\
\hline 2012 & $9.29 \%$ & $1.24 \%$ & $3.41 \%$ & $8.36 \%$ & $3.72 \%$ & $0.31 \%$ & $0.00 \%$ & $3.41 \%$ \\
\hline 2013 & $13.00 \%$ & $0.45 \%$ & $0.90 \%$ & $11.21 \%$ & $4.48 \%$ & $0.00 \%$ & $0.00 \%$ & $1.79 \%$ \\
\hline 2014 & $12.50 \%$ & $0.82 \%$ & $2.45 \%$ & $11.14 \%$ & $0.27 \%$ & $0.27 \%$ & $0.00 \%$ & $3.53 \%$ \\
\hline 2015 & $17.17 \%$ & $1.30 \%$ & $1.96 \%$ & $15.43 \%$ & $1.09 \%$ & $0.00 \%$ & $0.00 \%$ & $2.83 \%$ \\
\hline 2016 & $7.29 \%$ & $0.63 \%$ & $1.88 \%$ & $14.79 \%$ & $0.21 \%$ & $0.00 \%$ & $0.42 \%$ & $3.13 \%$ \\
\hline 2017 & $16.42 \%$ & $0.29 \%$ & $1.76 \%$ & $15.54 \%$ & $0.00 \%$ & $0.59 \%$ & $0.00 \%$ & $5.28 \%$ \\
\hline 10-year average & $11.11 \%$ & $0.67 \%$ & $1.50 \%$ & $10.85 \%$ & $2.19 \%$ & $0.20 \%$ & $0.37 \%$ & $2.21 \%$ \\
\hline $\mathrm{SD} \pm$ & $3.65 \%$ & $0.40 \%$ & $1.04 \%$ & $3.36 \%$ & $2.10 \%$ & $0.24 \%$ & $0.81 \%$ & $1.91 \%$ \\
\hline
\end{tabular}

*Represents FOX resistant and PBP2' latex agglutination-positive isolates.

$\mathrm{ERI}=$ erythromycin, $\mathrm{CHL}=$ chloramphenicol, CIP = ciprofloxacin, $\mathrm{CLI}=$ clindamycin, $\mathrm{DOX}=$ doxycycline, GEN = gentamicin, $\mathrm{SXT}=$ sulfamethoxazole/trimethoprim, MRSA = methicillin-resistant $S$. aureus, $S D=$ standard deviation.

Values in boldface represent peak resistance levels. 
scant data available in the literature regarding the susceptibility patterns of $S$. aureus isolates from gynecological samples, and therefore additional data are necessary to provide a comprehensive picture of resistance trends $(15,22,27-28)$. As a general rule, the susceptibility patterns were advantageous, and the resistance rates were below $20 \%$ in the case of every antibiotic tested. However, a slow and steady increase in resistance levels could be observed (corresponding to MRSA isolates, which are resistant to all $\beta$-lactam antibiotics, apart from fifth-generation cephalosporins), especially since 2012, when the first MRSA isolate was reported (22, 29-30). In a similar study in the same geographical region and time period (2008-2017), the resistance trends of $S$. aureus were described from male STI samples; more than $98 \%$ of isolates were methicillin-susceptible, and the highest levels of resistance were detected against macrolides (15-28\%) and clindamycin (13-30\%) (31). S. aureus as a urinary pathogen was also characterized in the southern part of Hungary: in these studies, 9.8 to $11.6 \%$ of isolates were MRSA between 2013 and 2017, and a numerically increasing tendency was observed (unpublished results). Based on the results of the European Antimicrobial Resistance Surveillance Network (EARS-Net), the European average for the percentage of MRSA was $16.9 \%$, showing a decreasing tendency with large inter-country variation (1.0-44.0\%) (32). Regarding Hungary, MRSA levels have been over $20 \%$ since the 2010 , and were fluctuating between 21 and $27 \%$ in the last 3 years of surveillance (2014-2017) $(32,33)$. Of course, the resistance situation is not as dramatic in the $S$. aureus isolates from vaginal samples (with the highest levels of MRSA detected around 5\%) compared to invasive isolates; nevertheless, the obvious increase in resistance should be noted.

There is no generally accepted clinical strategy for therapy for $\mathrm{AV}$; however, several proposals have been published. The distinction between $\mathrm{AV}$ and $\mathrm{BV}$ is crucial for the choice of appropriate therapy (3-13). Metronidazole has no effect on AV, unlike in BV and Trichomonas vaginalis infections, for which this drug is routinely used (6-9). Clindamycin may be considered as a valid therapeutic option (especially in pregnancy), whereas fluoroquinolones are recommended in non-pregnant females because their effect on the vaginal microbiota (Lactobacillus) seems to be minimal (3-13). However, antibiotic therapy should be complemented if the inflammation (topical steroids) or atrophy (estrogen) is pronounced. In addition, the use of vaginal probiotics should also be encouraged. Regarding the use of estrogen, in some patient populations (breast cancer patients and postmenopausal women) its use is contraindicated; in these cases, a very low dose of local es- triol should be used in combination with probiotics (3-13). Based on our results, the therapy for these genitourinary infections will not be hindered by antibiotic resistance for now; however, close surveillance should be performed for $S$. aureus isolates from all anatomical sites to monitor the changes in resistance trends (22). On the other hand, macrolides and CLI should be used with caution, and, if available, only when susceptibility to these drugs has been verified.

Limitations of this study must be acknowledged. First, the design of the study is retrospective and we could not access the medical records of the individual patients affected by these infections. For this reason, the correlation between the existence of relevant risk factors and underlying illnesses (apart from age) and the isolation of $S$. aureus could not be assessed. There is a risk of selection bias because studies describing the prevalence of various infectious diseases and resistance trends are mainly from tertiary-care centers, which generally correspond to patients with more severe conditions or underlying illnesses. Finally, a molecular characterization of the resistance determinants in the individual isolates was not performed (i.e., mecA or mecC genes), only to the extent of FOX resistance and latex agglutination tests.

\section{Conclusions}

$\mathrm{AV}$ is a distinct form of vaginal inflammation from BV, predominantly caused by $S$. aureus, $S$. agalactiae, and members of the Enterobacteriaceae family, which are abundantly present in case of vaginal dysbiosis. Because $S$. aureus is a significant pathogen in $\mathrm{AV}$, the aim of this study was to evaluate its relevance and frequency in vaginal samples at our tertiary-care teaching hospital during a 10-year study period (2008-2017). The highest levels of resistance were associated with CLI and macrolides (around 10\%), whereas MRSA-levels were below 5\% up until 2017. Continuous monitoring of resistance trends and the introduction of antimicrobial stewardship is recommended in the therapy for AV.

\section{Acknowledgements}

The authors would like to thank Tünde Deák and Erika Karasz for their excellent laboratory assistance during the routine diagnostic work. Márió Gajdács was supported by the National Youth Excellence Scholarship (Grant Number NTP-NTFÖ-18-C-0225) and the ESCMID Mentorship and Observership Programme.

\section{References}

1. Mendling W. Vaginal microbiota. Adv Exp Med Biol. 2019;902:83-93.

2. Martin DH. The microbiota of the vagina and its influence on women's health and disease. Am J Med Sci. 2012;343:2-9.

3. Miller EA, Beasley DE, Dunn RR, Archie EA. Lactobacilli dominance and vaginal $\mathrm{pH}$ : why is the human vaginal microbiome unique? Front Microbiol. 2016;7:1936.

4. Vásquez A, Jakobsson T, Ahnré S, Forsum U, Molin G. Vaginal lactobacillus flora of healthy Swedish women. J Clin Microbiol. 2002;40:2746-9.

5. Boris S, Suárez JE, Vázquez F, Barbés C. Adherence of human vaginal lactobacilli to vaginal epithelial cells and interaction with uropathogens. Infect Immun. 1998;66:1985-9.

6. Zárate G, Nader-Marcias ME. Influence of probiotic vaginal lactobacilli on in vitro adhesion of urogenital pathogens to vaginal epithelial cells. Lett Appl Microbiol. 2006;43:174-80.

7. Larsen B, Monif GRG. Understanding the bacterial flora of the female genital tract. Clin Infect Dis. 2001;32:e67-e77.
8. Rose WA, McGowin CL, Spagnuolo RA, Eaves-Pyles TD, Popov VL, Pyles RB. Commensal bacteria modulate innate immune responses of vaginal epithelial cell multilayer cultures. PlosOne. 2012;7:e032728.

9. Smith WL, Hedges SR, Mordechai E, Adelson ME, Trama JP, Gygax SE, et al. Cervical and vaginal flora specimens are highly concordant with respect to bacterial vaginosis-associated organisms and commensal lactobacillus species in women of reproductive age. J Clin Microbiol. 2014;52:3078-81.

10. Spence D, Melville C. Vaginal discharge. BMJ. 2007;335:1147-51.

11. Mitchell H. Vaginal discharge-causes, diagnosis, and treatment. BMJ. 2004; 328:1306.

12. Donders GGG, Bellen G, Grinceviciene S, Ruban K, Vieriea-Baptista P. Aerobic vaginitis: no longer a stranger. Res Microbiol. 2017;168:845-58.

13. Sun $X$, Qui $H$, Jin Y. Highly efficient treatment of aerobic vaginitis with simple acidic buffered gels: the importance of $\mathrm{pH}$ and buffers on the microenvironment of vaginas. Int J Pharmaceutics. 2017;52:175-82. 
14. Bagnall P, Rizollo D. Bacterial vaginosis-a practical review. JAAPA 2017;30:1521.

15. Sangeetha KT, Golia S, Vasudha CL. A study of aerobic bacterial pathogens associated with vaginitis in reproductive age group women (15-45 years) and their sensitivity pattern. Int J Med Sci. 2015;3:2268-73.

16. Mastromarino P, Vitali B, Mosca L. Bacterial vaginosis: a review on clinical trials with probiotics. New Microbiol. 2013;36:229-38.

17. Geng N, Wu W, Fan A, Han C, Wnag C, Wang Y, et al Analysis of the risk factors for aerobic vaginitis: a case-control study. Gynecol Obstetric Invest. 2016;81: 148-54.

18. Wen A, Srinivasan U, Goldberg D, Owen J, Marrs CF, Misha D, et al. Selected vaginal bacteria and risk of preterm birth: an ecological perspective. I Infect Dis. 2014;209:1087-94.

19. Fettweis JM, Serrano MG, Brooks JP, Edwards DJ, Girerd PH, Parikh HI, et al. The vaginal microbiome and preterm birth. Nature Med. 2019; 25:1012-21.

20. Ling Z, Liu X, Luo Y, Wu X, Yuan L, Tong X, et al. Associations between vaginal pathogenic community and bacterial vaginosis in Chinese reproductive-age women. Plos One. 2013;8:e76589.

21. Gajdács M, Urbán E. Epidemiological trends and resistance associated with Stenotrophomonas maltophilia bacteremia: a 10-year retrospective cohort study in a tertiary-care hospital in Hungary. Diseases. 2019;7:41.

22. Gajdács $M$. The continuing threat of methicillin-resistant Staphylococcus aureus. Antibiotics. 2019;8:52.

23. Hospital bed count and patient turnover report 2017. National Health Insurance Fund of Hungary; [cited 2019 May 8]. Available from: http://www.neak.gov.hu/ felso_menu/szakmai_oldalak/publikus_forgalmi_adatok/gyogyito_megelozo_ forgalmi_adat/fekvobeteg_szakellatas/korhazi_agyszam.html.

24. Gajdács M, Spengler G, Urbán E. Identification and antimicrobial susceptibility testing of anaerobic bacteria: Rubik's cube of clinical microbiology? Antibiotics. 2017;6:25.
25. Nagy E, Becker S, Kostrzewa M, Barta N, Urbán E. The value of MALDI-TOF MS for the identification of clinically relevant anaerobic bacteria in routine laboratories. J Med Microbiol. 2012;61:1393-400.

26. Ábrók M, Lázár A, Szécsényi M, Deák, J, Urbán, E. Combination of MALDI-TOF MS and PBP2' latex agglutination assay for rapid MRSA detection. J Microbiol Methods. 2018;144:122-4.

27. Guinan ME, Dan BB, Guidotti RJ, Reingold AL, Schmid GP, Bettoli EJ, Lossick JG, Shands KN, Kramer MA, Hargrett NT, Anderson RL, Broome CV. Vaginal colonization with Staphylococcus aureus in healthy women: a review of four studies. Ann Intern Med. 1982;96:944-7.

28. Mumtaz S, Ahmad M, Aftab I, Akhtar N, ul Hassan M, Hamid A. Aerobic vaginal pathogens and their sensitivity pattern. J Ayub Med Coll Abbottabad. 2008;20: $113-7$.

29. Gajdács M. The concept of an ideal antibiotic: implications for drug design. Molecules. 2019;24:892.

30. Gajdács M. [Extra deaths due to pandrug resistant bacteria: a survey of the literature]. Egészségfejlesztés. 2019;60:31-6. Hungarian.

31. Gajdács M. [Epidemiology and susceptibility patters of Staphylococcus aureus isolates from STI samples of male patients (2008-2017)]. Magyar Urológia. 2019;31:66-8. Hungarian.

32. European Antimicrobial Resistance Surveillance Network (EARS-Net). [cited 2019 Jul 11]. Available from: https://ecdc.europa.eu/en/about-us/partnershipsand-networks/disease-and-laboratory-networks/ears-net.

33. Conceição T, Aires-de-Sousa M, Füzi M, Tóth A, Pászti J, Ungvári E, et al. Replacement of methicillin-resistant Staphylococcus aureus clones in Hungary over time: a 10-year surveillance study. Clin Microbiol Infect. 2007;13:971-9. 